\title{
An investigation into the effect of magnetic resonance imaging (MRI) echo time spacing and number of echoes on the sensitivity and dose resolution of PAGATUG polymer-gel dosimeter
}

\author{
S.M. Abtahi ${ }^{*}$, R. Jafari Khalilabadi², S. Aftabi3 \\ 1Imam Khomeini International University, Physics Department, P. O. Box 34149-16818, Qazvin, Iran. \\ ${ }^{2}$ Shahid Beheshti University of Medical Sciences, Radiation Technology Department, Tehran, Iran. \\ ${ }^{3}$ Young Researchers and Elite Club, Rasht Branch, Islamic Azad University, Rasht, Iran
}

\section{- Original article}

*Corresponding author:
Dr. S.M. Abtahi,
Fax: +98 2833780040
E-mail:
$\quad$ smabtahi2007@gmail.com
Revised: March 2016
Accepted: May 2016
Int. J. Radiat. Res., April 2017;
15(2): 185-196
DOI: 10.18869/acadpub.ijrr.15.2.185

\begin{abstract}
Background: There are various methods to read out responses of a polymergel dosimeter, among which the Magnetic Resonance Imaging (MRI) technique is the most common one. Optimizing imaging protocols can have significant effect on the sensitivity and the dose resolution of polymer gel dosimeters. This study has investigated the effects of the number of echoes (NOE) and the echo time spacing (ES) parameters on the response of the PAGATUG polymer gel dosimeter. Materials and Methods: The polymer gel dosimeters were produced under the normal atmospheric condition, then irradiated using a ${ }^{60} \mathrm{Co}$ clinical radiation source. The polymer gel dosimeters were imaged using MRI technique with different imaging protocols. Afterwards, the effects of the ES and the NOE on the $R_{2}$-sensitivity and the dose resolution of PAGATUG polymer gel dosimeter have been investigated. Results: Results showed that the sensitivity decreased with increasing NOE. However, it was found that the resolution got better with increasing NOE. Furthermore, the investigation of signal intensity decay curve showed that the R2 values in NOE less than 5 was less reliable. According to the results, no significant difference were found between the sensitivities of the polymer gel dosimeters which were imaged with different ESs, except for ES=22 ms. However, dose resolution suffered from ES increasing. Conclusion: Dosimetric parameters of PAGATUG polymer gel dosimeter reaches to their optimum values by imaging with $E S=25 \mathrm{~ms}$ and $\mathrm{NOE}=10$.
\end{abstract}

Keywords: Polymer gel dosimeter; PAGATUG; MRI; Number of echoes; Echo time spacing.

\section{INTRODUCTION}

The aim of modern radiation therapy is to deliver a high dose to a tumor as well as preventing damage to healthy tissues (1) . Any errors in radiotherapy of cancerous tissues, especially when they are adjacent to vital organs, create additional risk. Due to such errors not only the cancerous tissue do not receive proper dose, but also vital organs are irradiated, consequently irradiation causes serious damages.
Therefore, it is necessary to carefully investigate the dose distribution before treatment to make sure that besides receiving the dose by all parts of the tumor, normal tissues and more importantly vital organs are safely protected (2). Conventionally, to achieve this objective, a series of dosimeters are placed inside a phantom, and are irradiated in real treatment conditions (2). However, using these conventional methods lead to the loss of spatial resolution. Polymer gel dosimeters, in addition to their 
three-dimensional nature, create highly spatial resolution $(3,4)$. Polymer gel dosimeters have been shown to be useful tools in order tomeasure dose distributions in special applications of radiotherapy, such as intensity-modulated radiotherapy and stereotactic radiosurgery $(5,6)$. Gel dosimeters have also been applied to proton beams $(7,8)$, high-energy carbon ion beams (9) and neutron beams (10-12) . Polymer gel dosimeters, in fact, are monomers that are uniformly distributed in a gelatin matrix (13) . The interaction of the radiation with water molecules splits them and the radiolytic products initiate the polymerization reaction. The amount of polymer formed at any point is proportional to the absorbed dose at that point (14) . Changing monomers into polymers causes changes in dosimeter's physical and chemical characteristics that are measurable with different methods, such as optical Computed Tomography (optical CT) (15-17), X-ray Computed Tomography (X-ray CT) (18,19), ultrasound (20), and MRI (21,22). The most common method is the investigation of changes in spin-spin relaxation rate $\left(R_{2}\right)$ due to irradiation $(23,24)$. Optimization of imaging techniques can have significant influences on improving sensitivity and resolution of polymer gel dosimeters (25). Baldock et al. investigated the effect of the echo time spacing on dose resolution of the polyacrylamide polymer gel dosimeter (PAG) (26). They concluded that at a certain $\mathrm{T}_{2}$ range, the dose resolution could be optimized by proper ES selection. In another study Baustert et al. investigated the effect of different MRI sequences on the accuracy of PAG polymer gel dosimeter (27). They suggested MSE sequence in order to improve accuracy. Other studies have also been conducted on impact of imaging parameters on dosimeter response (28-30). However, all of these studies have been performed on the effect of MR parameters on hypoxic polymer gel dosimeters, and effect of MR parameters on normoxic polymer gel dosimeters has not been investigated yet. The aim of this study therefore was to evaluate the efficacies of optimization of two important imaging parameters on the response of a normoxic polymer gel dosimeter.

Recently, a polymer gel dosimeter with interesting properties, including a significant increase in sensitivity, has been reported by Abtahi et al. (31). This polymer gel dosimeter was named PAGATUG (Poly Acrylamide, Gelatin And Tetrakis (PAGAT), Urea, Glucose). The PAGATUG polymer gel dosimeter is made by adding urea and glucose to the well-known PAGAT polymer gel dosimeter compounds. Two important parameters which affect the sensitivity and the dose resolution are number of echoes (NOE) and echo time spacing (ES). In this study, the effects of the ES as well as the NOE on the PAGATUG polymer gel dosimeter response have been investigated. Furthermore, in this study, a new parameter named NAN (Not A Number) which is the number of loss data in R2 map has been introduced. The effects of ES and NOE variations on NANs have also been investigated.

\section{MATERIALS AND METHODS}

\section{Polymer gel dosimeter production}

The PAGATUG polymer gel dosimeter has been made based on the formulation developed by Abtahi et al. (31). Chemical components used to make the PAGATUG polymer gel dosimeter are as follows: Ultra-pure water with High-Performance Liquid Chromatography (HPLC) grade (made by Direct-Q 3 UV water purification system, Millipore, France), Gelatin (5\% mass fraction $(\mathrm{w} / \mathrm{w})$ ) (porcine skin, type $\mathrm{A}$, 300 Bloom, Sigma-Aldrich, USA), Acrylamide $(3 \% \mathrm{w} / \mathrm{w})$ (Plus One Acrylamide Page, $100 \%$, GE Healthcare UK Ltd), N,N'- methylene diacrylamide (bis) $(3 \% \mathrm{w} / \mathrm{w})$ (electrophoresis grade, Merck, Germany), Tetrakis hydroxymethyl phosphonium chloride (THPC) (5 mM) (\% 80 solution in water, Sigma Aldrich, USA), Hydroquinone (0.01 mM) (Merck, Germany), Glucose-D (+) $(8.5 \% \mathrm{w} / \mathrm{w})$ (anhydrous, for bio-chemistry, Merck, Germany) and urea (3\% w/w) (Urea crystal, Ultrapure, Merck, Germany).

The PAGATUG polymer gel dosimeter has been made under the hood in the normal atmosphere condition. The manufacturing meth- 
od was the same as the report of Abtahi etal. (31). Glucose and urea were first completely dissolved in $90 \%$ of water at room temperature, and gelatin was then added. The mixture was heated to $48^{\circ} \mathrm{C}$ and stirred with a magnetic bar until gelatin was completely melted. Bis was dissolved at $48{ }^{\circ} \mathrm{C}$ and then the heater was turned off. Acrylamide was added when the mixture was cooled to about $37^{\circ} \mathrm{C}$. A solution of the antioxidant THPC and HQ were prepared with the remaining $10 \%$ of the water, and added to the solution at about $35{ }^{\circ} \mathrm{C}$. The polymer gel dosimeter was then transferred into $5 \mathrm{ml}$ cuvettes, sealed with parafilm, and stored in a refrigerator, at $5^{\circ} \mathrm{C}$, until irradiation.

\section{Irradiation}

The irradiation took place a day after the polymer gel dosimeter production using ${ }^{60} \mathrm{Co}$ (Theratronics, Theratron 780-C, Canada). Cuvettes filled with the polymer gel dosimeter were placed in a water phantom at $5 \mathrm{~cm}$ depth to satisfy scattering conditions. The irradiation was performed at a field size of $20 \times 20 \mathrm{~cm}^{2}$, a source surface distance (SSD) of $75 \mathrm{~cm}$, and a dose rate of $1.06 \mathrm{~Gy} \cdot \mathrm{min}^{-1}$ in the center of the cuvettes. The delivered doses were 2, 5, 7, 10, 13, 16, 17, 20 and 25 Gray (Gy), respectively. Two cuvettes were not irradiated as control cuvettes. The delivered doses to the cuvettes were calibrated using an ionization chamber (Farmer type, 0.6 $\mathrm{cm}^{3}$, PTW, Germany) according to the TRS 398 protocol reported by the International Atomic Energy Agency (32). The cuvettes were placed in the water bath at the irradiation room temperature for at least 2 hours before irradiation in order to become isothermal with the environment. The water bath and room temperature were measured both equal to $22 \pm 0.5^{\circ} \mathrm{C}$.

\section{Response read out}

In order to read-out the PAGATUG polymer gel dosimeter, magnetic resonance imaging (MRI) was used. For this purpose, after irradiation, the polymer gel dosimeters were transferred to the MRI scanning room. After being isothermal with the environment, the polymer gel dosimeter cuvettes were imaged by means of MRI (Siemens, Avanto, Germany) with the magnetic field strength of 1.5 Tesla. All of the imaging tests were performed at least 45 days after the irradiation. Passing this time guaranteed that both the polymerization reaction due to long-lived radicals and the gelation-process did not affected the polymer gel dosimeter (32). Both of these processes could introduce instabilities of $\mathrm{R}_{2}$ over the time. In order to perform imaging, the head coil was used because signal-to-noise ratio (SNR) of the head coil is greater than the body coil (22).

A slice-selective multiple spin-echo sequence with a Carr-Purcell-Meiboom-Gill (CPMG) pulse scheme was applied. The imaging tests were performed in two different rounds. In the first round, ES was fixed and NOE was changed. Second round was performed with a fixed NOE and variable ES.

In the first round, the NOEs of $3,5,7,8,9$, $10,11,12,14,15$ and 16 were selected with the ES of $22 \mathrm{~ms}$. In the second round, the NOE of 6 and the ESs of 22, 25, 34, 41 and $50 \mathrm{~ms}$ were selected. Other parameters were a repetition time (TR) of $4000 \mathrm{~ms}$, a matrix size (MS) of $512 \times 512$, a field of view (FOV) of $256 \mathrm{~mm}$, and a bandwidth (BW) of $100 \mathrm{~Hz}$.

What can be obtained from multi spin-echo imaging with MRI system is a set of signal intensity (SI) images. Using the signal intensity images in the different echo times and Equation 1 to the SI-time data, the related relaxation $\left(\mathrm{R}_{2}\right)$ rate can be obtained

$$
s=s_{0} e^{\left(-R_{2}, T E\right)}
$$

Where TE is the echo time, $s$ is the signal intensity at $\mathrm{TE}$, and $\mathrm{s}_{0}$ is the signal intensity at $\mathrm{TE}=0$. Fitting process has been performed using a method explained previously by De Deen et al. (33). The $\mathrm{R}_{2}$ of each pixel has been obtained. These values are finally presented as a $R_{2}$ map. A typical R2 map of the PAGATUG polymer gel dosimeter has been shown in figure 1 . To obtain the $R_{2}$ map of the signal intensity images, an M-file has been written in MATLAB (Mathworks, Natic, MA).

Int. J. Radiat. Res., Vol. 15 No. 2, April 2017 


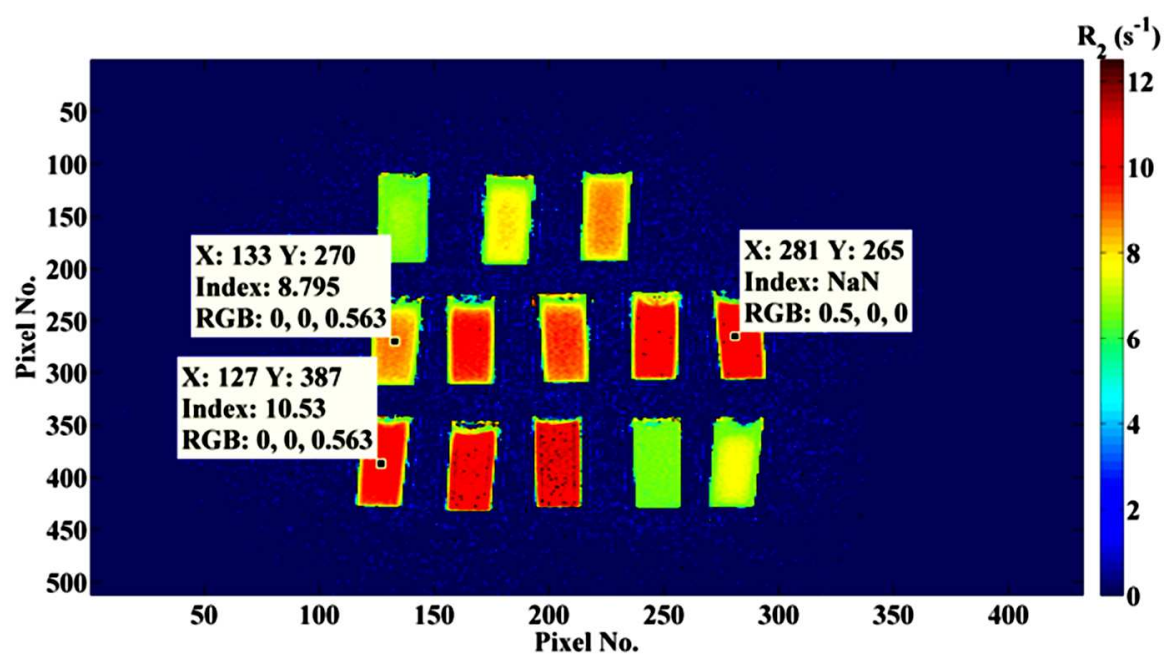

Figure 1. The $R_{2}$ map of the PAGATUG polymer gel dosimeter obtained from a sequential signal intensity with ES=22ms. Data cursors on some points are exhibited. The corresponding R2 values of some pixels are represented as "index" value.

\section{The dose resolution calculation}

After extracting the $\mathrm{R}_{2}$ map from the signal intensity images, the average amount of $\mathrm{R}_{2}$ for each region of interest (ROI) was attributed to the absorbed dose in that ROI. To plot the dose- $\mathrm{R}_{2}$ curve, a bi-exponential function was fitted to the dose- $\mathrm{R}_{2}$ data. R-square and adjusted $\mathrm{R}$-square values were calculated to evaluate the goodness of the fit (34). However, the response of the polymer gel dosimeter has been investigated for doses beyond the inhibition region. The $R_{2}$ amounts of the irradiated cuvettes indicate lack of response for doses up to 2Gy. This unresponsive area is attributed to the oxygen permeability which inhibits the radiation induced polymerization reaction (35). Many features of a polymer gel dosimeter could be obtained from its dose- $\mathrm{R}_{2}$ curve. The dose- $\mathrm{R}_{2}$ curve also known as calibration curve. The slop of the dose- $\mathrm{R}_{2}$ curve indicates the dosimeter sensitivity whose unit is $\mathrm{Gy}^{-1} \mathrm{~s}^{-1}$. Another important feature derived from the calibration curve is the minimum separation between two distinguishable doses called dose resolution. The dose resolution is calculated using equation 2 (25).

$$
D_{\Delta}^{p}=k_{p} \sqrt{2} \frac{\sigma_{R_{2}}}{\alpha}
$$

Where $k_{p}$ is the coverage factor, $\sigma R_{2}$ is the standard deviation of the $\mathrm{R}_{2}$ in ROI and $\alpha$ is the slope of the Dose- $R_{2}$ curve, or the dosimeter sensitivity. The values of coverage factors of $67.27 \%, 90 \%, 95 \%, 95.45 \%, 99 \%$ and $99.73 \%$ are $1.000,1.645,1.96,2.000,2.576$ and 3.000 respectively (36). In a many point method (33), which has been used in this study for extracting R2 from signal intensity images, standard deviation in R2 map is theoretically obtained using equation 3 :

$$
\approx \frac{\sigma_{R_{2}}}{\approx} \frac{\sigma_{S}}{S_{0} N\left(N^{2}-1\right) \Delta T E} \sqrt{\left.(N-1)^{2}\left(1-\lambda^{N+2}\right)+2\left(N^{2}-3\right)\left(\lambda^{N}-1\right) \lambda-(N+1)^{2}\left(\lambda^{N-2}-1\right)^{2}\right)^{2} /(1-\lambda)^{3}}
$$

Where $\lambda=\mathrm{e}^{2 \mathrm{R}_{2} \Delta \mathrm{TE}}, \Delta \mathrm{TE}$ is the ES and $\mathrm{S}_{0}$ is the maximum signal intensity. $\sigma s$ Is the thermal noise of the signal $S$ and can be considered independent from the echo time. In this expression $\mathrm{N}$ is the NOE.

\section{RESULTS}

\section{The effects of the NOE}

The $R_{2}$ changes as a function of the imaging NOE have been shown in figure 2 . The $R_{2}$ values in the low NOE are strongly dependent on the imaging NOE. However, in the NOE over than 8, the $R_{2}$ values are independent from the imaging NOE with good approximation. The results have illustrated less dependence of $\Delta \mathrm{R}_{2}\left(=\mathrm{R}_{2}-\mathrm{R}_{2,0}\right)$ o the variation of the NOE. However, this dependence at low doses was still significant. 
As can be obtained from figure 2, a bi-exponential equation can be fitted to the dose $-\Delta R_{2}$ data. As it has been shown in figure 3 , the sensitivity of the PAGATUG dosimeter has changed with the NOE. It was found that the sensitivity decrease with increasing the NOE. However, the investigation of signal intensity decay curve showed that the R2 values in NOE less than 5 was less reliable.

Figure 4 (a) and (b) shows signal intensity (normalized to 1) decay curve for a typical pixel for NOE of 16 and 5, respectively. The R-square and adjusted R-square for both curves are more than 0.995. In a previous research it has been shown that there should be enough echoes to cover the exponential signal decay until it reaches the baseline (23). Therefore, decreasing the NOE in order to increase the sensitivity is not advised. For NOEs more than 6, statistical analyses (37) showed that the variation in sensitivity was not significant. As can be seen in figure 4, signals related to the first, second and third echoes deviate from the exponential decay regime. Also, for echo times more than $286 \mathrm{~ms}$ (NOE=13), the signal intensities deviate from exponential fitting. This deviation at the tail of the curve was attributed to the effect of background noise which is compatible with the signal intensity.
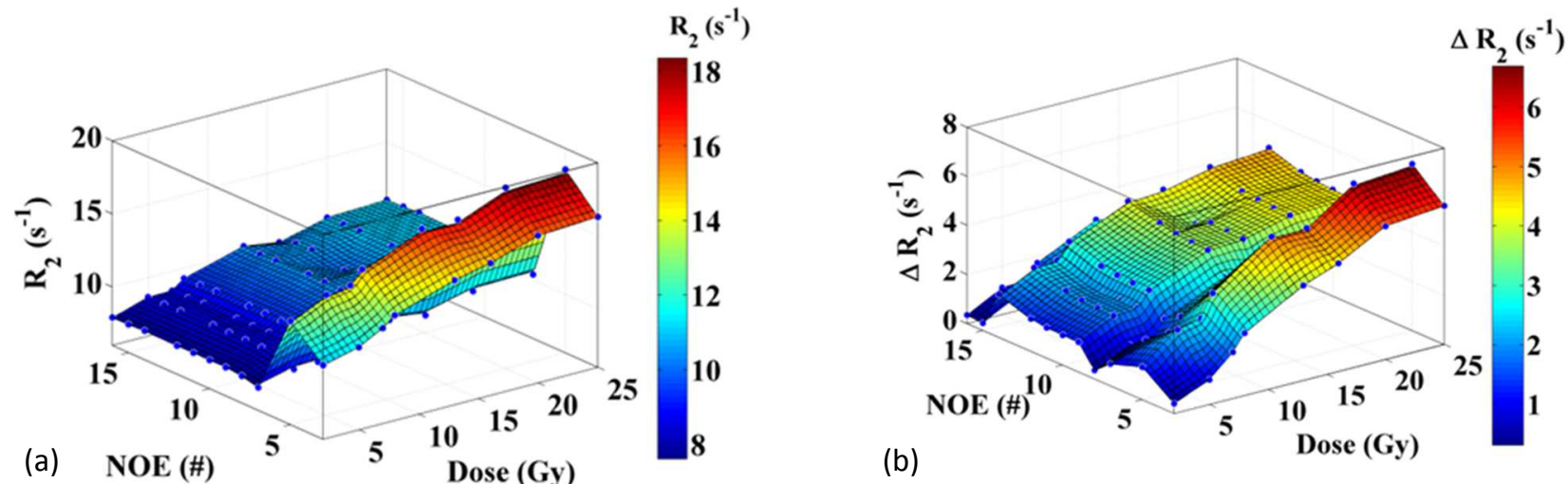

Figure 2. (A) The $R_{2} s$ as a function of the absorbed dose and the imaging NOE for the irradiated PAGATUG dosimeter. (B) $\Delta R_{2}$ $\left(=R_{2}-R_{2,0}\right)$ as a function of the absorbed dose and the imaging NOE.

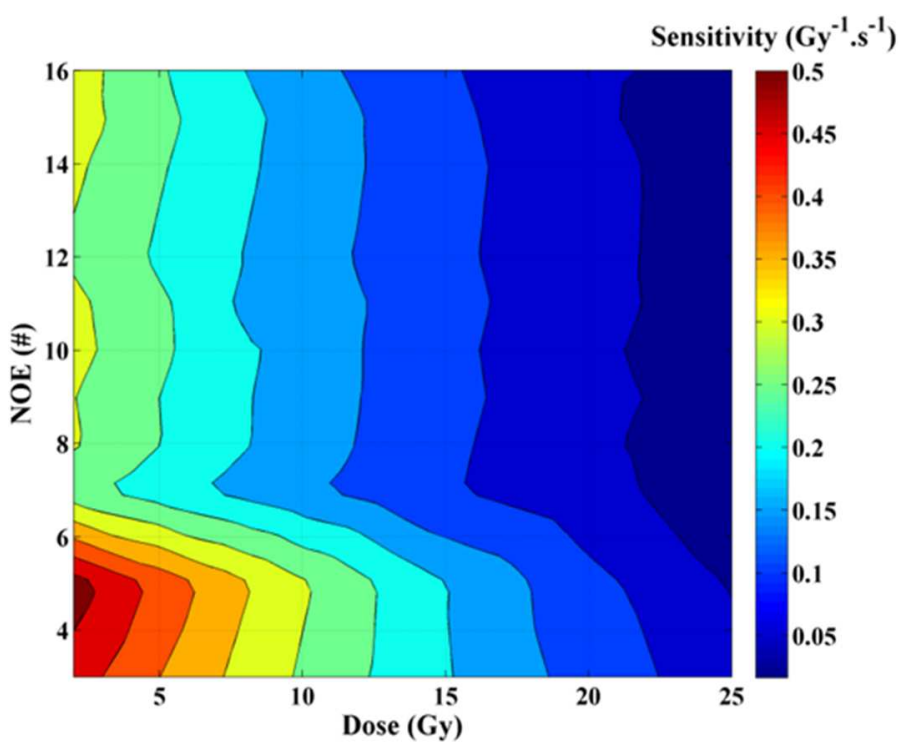

Figure 3. Variation of the sensitivity of the PAGATUG as a function of the NOE and the absorbed dose. The ES of $22 \mathrm{~ms}$ has been chosen for imaging tests. 

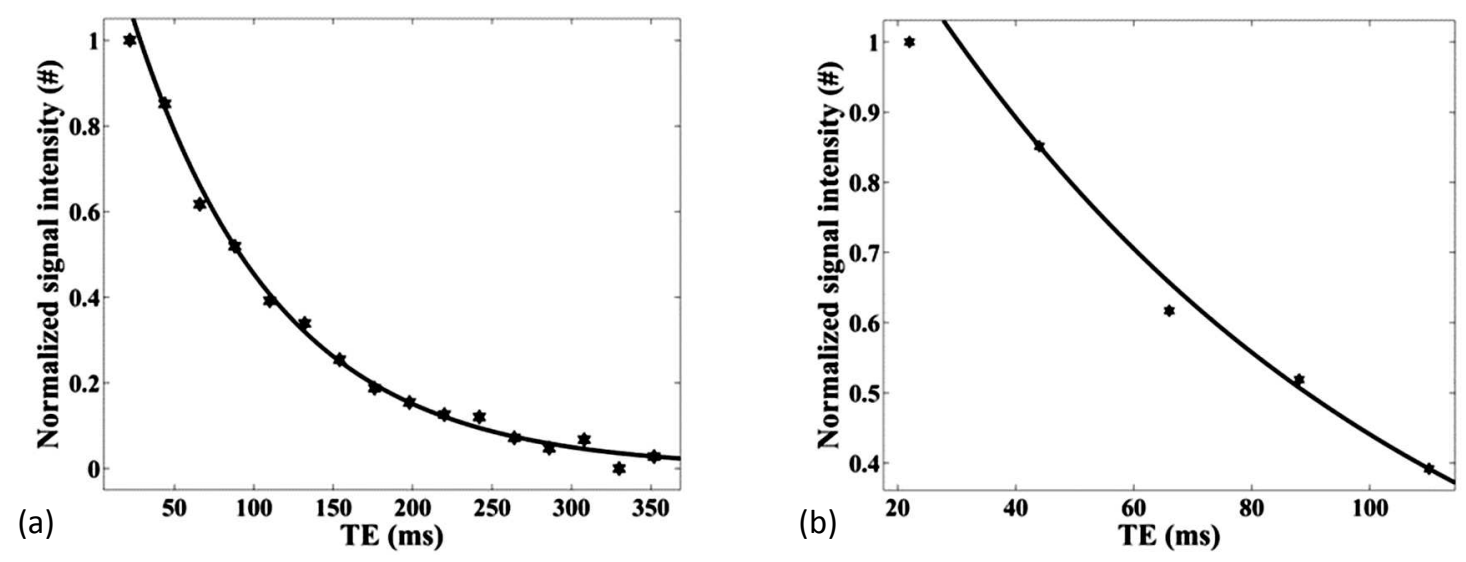

Figure 4. Signal intensity decay for a typical pixel for (a) NOE of 16 and (b) NOE of 5.

Figure 5 shows the dose resolution as a function of the NOE at different doses for PAGATUG polymer gel dosimeter. As can be seen in figure 5 , the resolution value decreases with the increase of NOE (the resolution gets better). This result could be interpreted by Equation 3 . In Equation 3 since $\lambda$ is a relatively small quantity, the standard deviation in R2 map is proportional with $1 / \mathrm{N}^{1.2}$. Equation 2 shows that an increase in the sensitivity in low NOE can relatively decrease the quantity of dose resolution. However, this increase has less effect in comparison with $\sigma \mathrm{R}_{2}$ decrease. As an example, for the PAGATUG polymer gel dosimeter with 5 Gy absorbed dose the sensitivities for the NOE of 5 and 15 are 0.44 $\mathrm{Gy}^{-1} \mathrm{~s}^{-1}$ and $0.26 \mathrm{~Gy}^{-1} \mathrm{~s}^{-1}$, respectively. However, related $\sigma \mathrm{R}_{2} \mathrm{~s}$ are $0.054 \mathrm{~s}^{-1}$ and $0.014 \mathrm{~s}^{-1}$, respectively. The results of this study show that the dose resolution is proportional to $1 / \mathrm{N}^{1.2}$. The reduction of power of denominator was attributed to the sensitivity decrease with NOE. In addition, referring to figure 4 results of figure 5 can be qualitatively justified. Dose-resolution is inversely proportional to the sensitivity. However, the dose resolution is directly proportional to the standard deviation in the R2 map. As can be seen in Figure 4 at low NOE which does not cover the exponential decay curve of the signal intensity, the R2 value deviates from its real value. This deviation increases the standard deviation in the R2 map which is dominant in comparison with the effect of increasing sensitivity. Therefore, the dose resolution quantity increases with NOE decreasing.

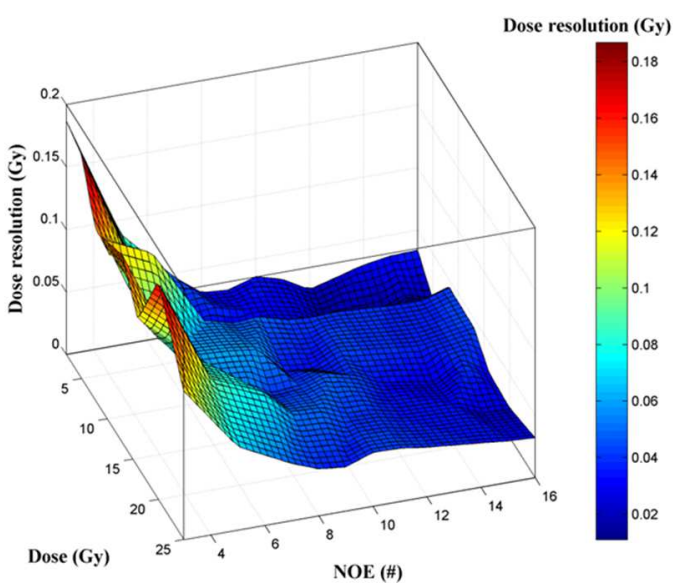

Figure 5. The dose resolution of the PAGATUG polymer gel dosimeter dosimeter at $68 \%$ level of confidence as a function of the NOE and the absorbed dose. The ES of $22 \mathrm{~ms}$ has been chosen for imaging tests. 
For some pixels in the R2 map, the exponential fitting to the Time-Signal intensity data results in unreasonable $\mathrm{R} 2$ values. These pixels were named NANs in this study. The number of NAN points changes by NOE in a R2 map. As an example, a NAN point in a typical R2 map have been represented in figure 1. Figure 6 shows the variation of NANs as a function of the NOE. At low NOE overestimation and underestimation in signal intensities cause deviation from the exponential signal decay; consequently, the number of NANs increases. However, with increasing of the NOE the number of NANs decreases. At the NOEs of about 10-12 there are sufficient echoes to cover the exponential signal decay. Therefore, the number of NANs reaches to its minimum. Afterward, the number of NANs increases with increasing NOE.
This NANs increase was attributed to the SNR reduction. The SNR reduction is a result of background sampling at time interval much longer than the length of $\mathrm{T} 2$. It has been previously shown that sampling at the time interval much longer than the length of $\mathrm{T} 2$ causes large uncertainties on R2 values and the accuracy of the R2 estimation suffers (23).

Fewer NANs provide fewer points that have lost their R2 information. Thus, fewer NANs are more desirable. As can be seen in Figure 6, there is a relatively broad minimum in the range of 10 to 12 echoes in the NAN-NOE graph.

\section{The effects of the ES}

The effect of the ES on the $\mathrm{R}_{2}$ and $\Delta \mathrm{R}_{2}$ at different doses has been demonstrated in figure 7.

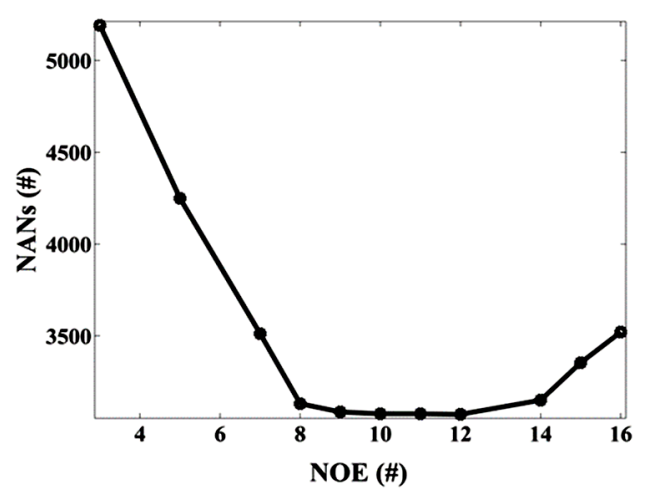

Figure 6. The variation of NANs in $R_{2}$ maps as a function of the NOE for the PAGATUG polymer gel dosimeter. The lines are plotted as a guidance and do not contain any physical information. The number of NANs has been considered in the total $R_{2}$ maps.
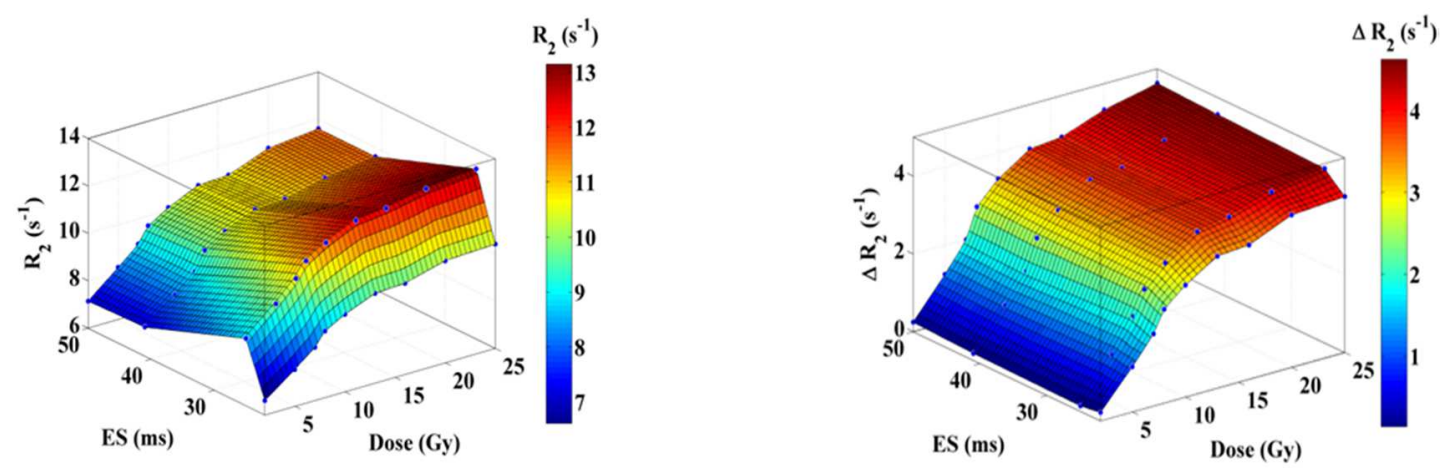

Figure 7. (a) The $R_{2}$ (b) and the $\Delta R_{2}$ as a function of the absorbed dose and the ES for the PAGATUG polymer gel dosimeter.

The effect of the ES on the R2 can be compared with the study of Baldock et al. (26). They compared the $\mathrm{R}_{2}$ values of irradiated PAG polymer gel dosimeter which was imaged with 191 the ESs of $12.5 \mathrm{~ms}$ and $50 \mathrm{~ms}$. They reported a $35 \%$ maximum difference in the R2s of the PAG imaged with $\mathrm{ES}=12.5 \mathrm{~ms}$ and $\mathrm{ES}=50 \mathrm{~ms}$. This maximum difference was related to the $2.5 \mathrm{~Gy}$ 
absorbed dose. The results of our study have shown the maximum difference of $19 \%$ between the polymer gel dosimeters imaged with $E S=25$ $\mathrm{ms}$ and $\mathrm{ES}=50 \mathrm{~ms}$. The different results of this study and the work of Baldock et al. may be attributed to the different integrants in formulations as well as different imaging ESs. The $\Delta \mathrm{R}_{2}$ investigation has shown the response independency from ES in a wide range of ESs. Therefore, it can be concluded that the ES variation affects the $R_{2}$ values; however, this effect is independent from the $\mathrm{R}_{2}$ values.

The dosimeter sensitivity as a function of the absorbed dose and the ES has been shown in figure 8. Statistical analysis (37) showed no significant differences in the sensitivities of the polymer gel dosimeters which were imaged with different ESs, except for ES=22 ms. Imaging with $\mathrm{ES}=22 \mathrm{~ms}$ resulted in significantly decrease in the sensitivity.

figure 9 shows the dose resolution $D_{\Delta}^{\rho}$ at $68 \%$ level of confidence for the PAGATUG polymer gel dosimeter as a function of the absorbed dose

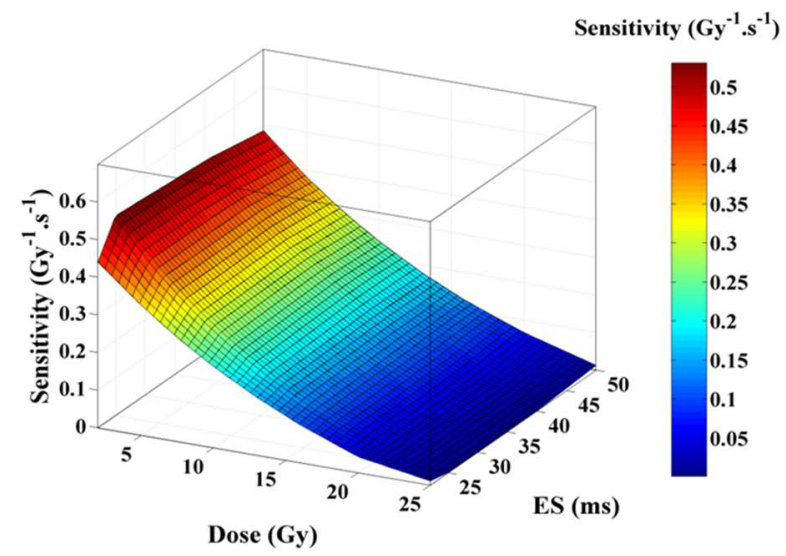

Figure 8. The sensitivity of the PAGATUG polymer gel dosimeter as a function of the absorbed dose and the ES.

Figure 10 shows the number of NANs, as a function of the absorbed dose and the ES. The NAN variations are not noticeable for ES less than $40 \mathrm{~ms}$, whereas the number of NANs significantly increases for ES $=50 \mathrm{~ms}$ which is attributed to the background sampling for imaging with $\mathrm{ES}=50 \mathrm{~ms}$.

The ES sensitivity curve (figure 8) illustrates Int. J. Radiat. Res., Vol. 15 No. 2, April 2017 and the ES. The results have showed that for all ESs, the $D_{\Delta}^{\rho}$ increases with increasing dose. This increase in $D_{\Delta}^{p}$ can be attributed to the sensitivity reduction with increasing absorbed dose. Previously, the bi-exponential variations of $\mathrm{R}_{2}$ as a function of the absorbed dose has been reported by De Deene et al. (38). The results of this study show that $D_{\Delta}^{p}$ increases with ES, which implies bad dose resolution. At lower doses, there is no significant increase in $D_{\Delta}^{p}$ with the ES increase. However, with the dose increase, the dose resolution degradation becomes noticeable. According to Equation 2, two parameters affect the dose resolution: sensitivity and standard deviation. As shown in figure 8, the sensitivity is independent from ES for an ES range of $25 \mathrm{~ms}-50 \mathrm{~ms}$. Therefore, the dose resolution degradation is attributed to the increase of the standard deviation which itself is the result of less precisely estimation of $\mathrm{R}_{2}$. On the other hand, this less precisely estimation is due to an increase in the space between two successive sampling.

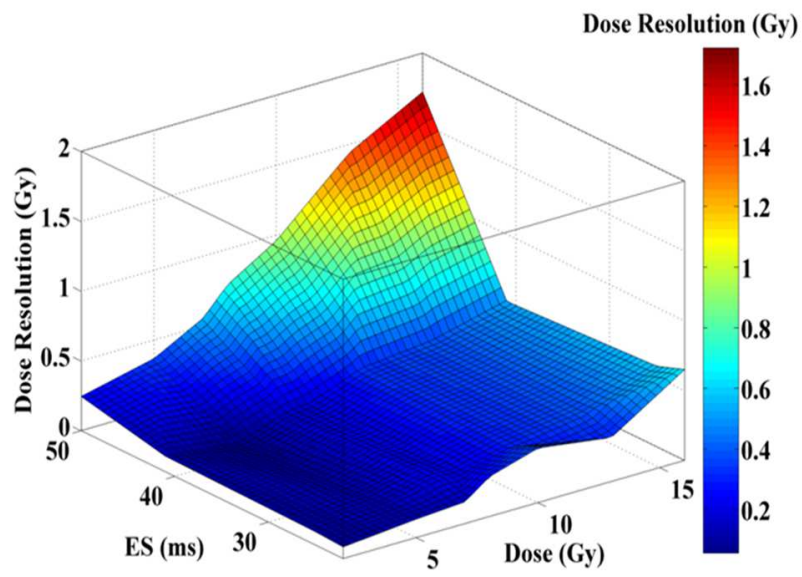

Figure 9. The dose resolutionat $\quad D_{a}^{p} \quad 68 \%$ level of confidence for the PAGATUG polymer gel dosimeter as a function of the absorbed dose and the ES.

that the sensitivity maximizes after ES $=25 \mathrm{~s}$. Statistical analyses (37) showed that with increasing ES, the sensitivity does not change significantly. Furthermore, statistical analysis showed that up to ES= $40 \mathrm{~ms}$, with ES increasing the dose resolution does not change significantly. The number of loss pixels (NANs) shows the minimum amount in ES $=40 \mathrm{~ms}$. 
However, the number of NANs in ES $=25 \mathrm{~ms}$ is only $2 \%$ more than its minimum amount. This difference does not result any disorder in the most practical situations. According to the above, in order to read out the PAGATUG with MRI technique, $\mathrm{ES}=25 \mathrm{~ms}$ is recommended.

Equation 4 demonstrates the $R_{2}$ exponential changes as a function of dose for $\mathrm{NOE}=10$ and $\mathrm{ES}=25$.

$R_{2}=11.27 e^{\left(1.824 \times 10^{-5} \times \text { dose }\right)}-5.844 e^{(-0.1186 \times \text { dose })}$

The R-square and the adjusted R-square for

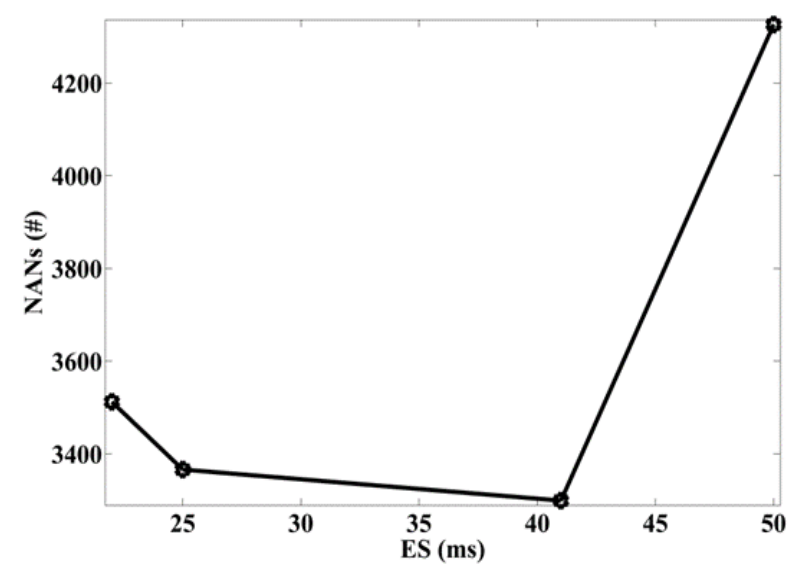

Figure 10. The number of NANs as a function of the ES. The number of NANs has been considered across each of the $R_{2}$ maps. The lines are plotted as a guidance and do not contain any physical information.

\section{DISCUSSION}

As reported by Abtahi etal. (31) the main advantage of the PAGATUG polymer gel dosimeter is its high sensitivity. However, in this study we have found that by optimizing the read -out parameters even higher sensitivity for the PAGATUG polymer gel dosimeter is achievable. In the polymer gel dosimetry literature different methodologies have been used to determine R2 quantitatively on a clinical MRI scanner. Azadbakht et al. used a modified multiple spin-echo pulse sequence with $E S=20 \mathrm{~ms}$ and NOE=32 on a Siemens Symphony MRI scanner (24). Gholami et al. used a multiple-spin-echo pulse sequence with $\mathrm{ES}=16.5 \mathrm{~ms}$ and $\mathrm{NOE}=32$ on a Siemens Avanto MRI scanner (39). Parwaie et al. used a multiple spin-echo sequence with $\mathrm{ES}=14$ fitting Equation 4 are equal to 0.9909 and 0.9863 which lead to an excellent fit. The linear portion of the response (dynamic range) of the PAGATUG polymer gel dosimeter is demonstrated in figure 11. Linear equation in dynamic range is given by Equation 5 .

$R_{2}=0.36 \times$ Dose +6.06

The R-square and the adjusted R-square for linear fitting are 0.999 and 0.993, respectively. The sensitivity of the PAGATUG polymer gel dosimeter is the slope of the linear portion (i. e. $0.36 \mathrm{~Gy}^{-1} \mathrm{~s}^{-1}$ ).

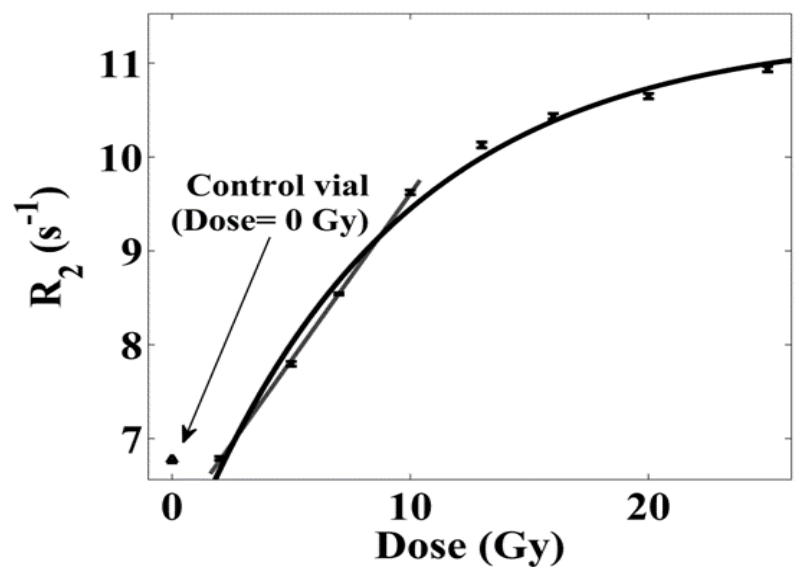

Figure 11. The dose- $R_{2}$ curves of the PAGATUG polymer gel dosimeter for $\mathrm{NOE}=10$ and $\mathrm{ES}=25$. $A$ linear model has been fitted to the dynamic range. The $\mathrm{R}_{2}$ value of the Control vial (vial with $0 \mathrm{~Gy}$ absorbed dose) has been shown.

ms and NOE=32 on a Siemens MRI scanner (40). However, the results of the presented study highlighted that the amounts of ES and NOE are important in determining the resulted dose-resolution, sensitivity, and the number of NANs in a R2 map.

Results show that the best NOE is the one in which sampling time is not more than average relaxation time. This finding is comparable with the work of Watanabe etal. which is about the effect of a variable echo-number on the response of a polymer gel dosimeter (23). They stated that if the echo time becomes much greater than T2, practically, the sampling will be performed from background signal intensity. This background sampling leads to the lower approximation in the $\mathrm{R}_{2}$, and an increase in the standard deviation in the ROI of the $\mathrm{R}_{2}$ map. Watanabe etal. only 
addressed to the issue of standard deviation in $\mathrm{R}_{2}$ maps, and the effect of NOE on other important parameters such as the sensitivity was not investigated.

It could be suggested that, there should be enough echoes to cover the exponential signal decay until it reaches the baseline or noise so that the majority of the decay is sampled. If a shorter ES is used, an increased NOE should, therefore, be used in order to collect a larger number of data.

Another aspect which is worth to discuss, is the results of NAN points. The creation of these points was attributed to the addition of noise to signal intensity images. NANs in R2 maps have been investigated in this study for the first time. This new quantity introduces loss points in a R2 map and also reduces spatial resolution. In previous studies, it has been shown that a noise in base images propagates to calculated maps (41). Furthermore, Scott et al. theoretically proved that the SNR of a MR image is a function of NOE (42). They showed a maximum amount for a distinguished NOE. The results of this investigation have verified findings of Scott et al. The results have shown a maximum value for SNR which has caused a minimum number of NAN points in a distinguished NOE.

The effect of the ES on the dosimeter response can be explained with the same mechanism as described for the effect of the NOE. Small ESs lead sampling to be perform more from the beginning of the signal intensity decay, and lead to overestimation or underestimation in the R2 values. Also, for the ESs more than a specified amount (its value is dependent on the NOE and T2), the tail of the signal intensity curve was sampled more significantly than the main body of the curve. This finding can be compared with that of previously reported by Wanatable et al. for BANG polymer gel dosimeter (23) . They found that if the last sampling be done at a time which is significantly more than T2, practically, the background signal is sampled which causes an inaccuracy in the $R_{2}$ evaluation. It is clear that this situation can happen with increasing ES. Thus, a random error occurs in the calculation of $\mathrm{R}_{2}$ value for different pixels that leads to an increase in the standard deviation and the
Furthermore, Equation 3 shows that is $\sigma \mathrm{R}_{2}$ a function of $\triangle T E$ so that the numerator of the fraction is an exponential function of $\Delta T E$. However, the denominator of fraction is a linear function of $\mathrm{R}_{2} \times \Delta \mathrm{TE}$. Hence, with the increase of $\Delta \mathrm{TE}$ a sharp increase in $\sigma \mathrm{R}_{2}$ especially for higher R2 values is expected. The experimental results of this study have confirmed the above mentioned theoretical inference.

According to the above descriptions, we can conclude that in order to perform sampling accurately, we all need to choose ES and NOE properly. Sampling at both low echo times and high echo times introduces random error in R2 estimation which can lead to destruction of the dose-resolution, the sensitivity and the spatial-resolution of the polymer gel dosimeter. Underestimation of first echo and overestimation of second echo are a common characteristic of the MRI pulse sequence based on Carr-Purcell Meiboom-Gill (CPMG). $D_{\Delta}^{p}$ This is related to realistic imperfect RF-pulses that result in a slice profile with shallow edges. These results in stimulated echoes make those slice profiles differ from echo to echo (43). Eventually (after 3 echoes) they reach a steady state. The theory behind this phenomenon was first documented by Fransson et al. in 1993 (44). Hence, it is recommended that the first two echoes be omitted from R2 estimation. However, this suggestion is more reliable than that was previously reported by Watanable et al. (23) . They only excluded the first echo signal from the R2 estimation processes. It seems that an increase in NOE leads to an increase in sampling, and as a result the $\mathrm{R} 2$ will be more accurately estimated. However, it should be noted that the tail of the signal intensity decay curve is not sampled. This result has been achieved by proper choice of the ES. In other words, the ES and the NOE should be selected in a way that there is enough sampling point to cover the signal intensity decay curve with the maximum sampled point before it reaches the background and the majority of the decay is sampled.

The sensitivity which obtained in this study for the PAGATUG polymer gel dosimeter was $16 \%$ more than what of previously reported for the PAGATUG polymer gel dosimeter (31). Furthermore, the dynamic range of $2 \mathrm{~Gy}-10 \mathrm{~Gy}$ in 
the present study has been improved in comparison with $3.5 \mathrm{~Gy}-10$ Gy which was previously reported (31).

\section{CONCLUSION}

Investigation of ES showed that R2-sensitivity, dose resolution and number of NAN points have their optimum values at $\mathrm{ES}=25 \mathrm{~ms}$. However, for NOE, it has been found that the $\mathrm{NOE}=10$ results better dose resolution and spatial resolution rather than other protocols.

In this study, it has been tried to achieve a minimum random error, and consequently maximum accuracy for the PAGATUG polymer gel dosimeter with optimizing NOE and ES parameters. Moreover, for further works, we suggest that one can study the PAGATUG polymer gel dosimeter read out by simultaneously optimizing random errors and systematic errors of MRI technique.

\section{ACKNOWLEDGEMENTS}

The authors acknowledge the Radiology Department of the Besat Hospital (Kerman, Iran) and the Radiotherapy Department of the Shohada -e-Tajrish Hospital (Tehran, Iran) for their kindly cooperation. We would like to acknowledge the Polymer Laboratory of the Faculty of Chemistry of Shahid Beheshti University.

\section{Conflicts of interest: Declared none.}

\section{REFERENCES}

1. De Deene Y, De Wagter C, Van Duyse B, Derycke S, De Neve W, Achten E (1998) Three-dimensional dosimetry using polymer gel and magnetic resonance imaging applied to the verification of conformal radiation therapy in head-and-neck cancer. Radiother Oncol, 48:283-91.

2. Khan FM (2003) The physics of radiation therapy. Lippincott Williams \& Wilkins, Philadelphia.

3. Ibbott GS (2004) Application of gel dosimetry. J Phys Conf Ser, 3:58-77.
4. Baldock C, Deene YD, Doran S, Ibbott G, Jirasek A, Lepage M, McAuley KB, Oldham M, Schreiner9 LJ (2010) Polymer gel dosimetry. Phys Med Biol, 55:R1-R63.

5. Keshtkar M, Takavar A, Zahmatkesh MH, Nedaie HA, Vaezzadeh A, Naderi M (2014) Three-dimensional gel dosimetry for dose volume histogram verification in compensator-based IMRT. Int J Radiat Res, 12(1):13-20.

6. Allahverdi Pourfallah $\mathrm{T}$, Allahverdi M, Riyahi Alam N, Ay MR, Zahmatkesh MH (2009) Verifying the accuracy of dose distribution in Gamma Knife unit in presence of inhomogeneities using PAGAT polymer gel dosimeter and MC simulation. Int J Radiat Res, 7(1):49-56.

7. Gustavsson H, Back SAJ, Medin J, Grusell E, Olsson LE (2004) Linear energy transfer dependence of a normoxic polymer gel dosimeter investigated using proton beam absorbed dose measurements. Phys Med Biol, 49:384755.

8. Zeidan OA, Sriprisan SI, Lopatiuk-Tirpak O, Kupelian PA, Meeks SL, Anderson MD, Hsi WC, Li Z, Palta JR, Maryanski MJ (2010) Dosimetric evaluation of a novel polymer gel dosimeter for proton therapy. Med Phys, 37(5):2145-52.

9. Ramm $U$, Weber $U$, Bock $M, K r$ "amer $M$, Bankamp $A$, Damrau M, Thilmann C, B"ottcher HD, Schad LR, Kraft G (2000) Three-dimensional BANGTM gel dosimetry in conformal carbon ion radiotherapy. Phys Med Biol, 45:N95N102.

10. Uusi-Simola J, Savolainen S, Kangasm"aki A, Heikkinen S (2003) Study of the relative dose-response of BANG-3R polymer gel dosimeters in epithermal neutron irradiation. Phys Med Biol, 48:2895-906.

11. Abtahi SM, Aghamiri SMR, Khalafi H, Rahmani F (2014) An investigation into the potential applicability of gel dosimeters for dosimetry in boron neutron capture therapy. Int $\mathrm{J}$ Radiat Res,12(2):139-49.

12. Abtahi SM, Zahmatkesh MH, Khalafi H (2015) Investigation of an improved MAA-based polymer gel for thermal neutron dosimetry. J Radioanal Nucl Chem, 307(2):855-68.

13. Maryanski MJ, Schulz RJ, Ibbott GS, Gatenby JC, Xie J, Horton D, Gore JC (1994) Magnetic resonance imaging of radiation dose distributions using a polymer-gel dosimeter. Phys Med Biol, 39:1437-55.

14. Deene YD, Vergote K, Claeys C, Wagter Cd (2006) The fundamental radiation properties of normoxic polymer gel dosimeters: a comparison between a methacrylic acid based gel and acrylamide based gels. Phys Med Biol, 51:653-73.

15. De Jeana $P$, Sendenb R, McAuleyb K, Rogersa M, Schreinera $L$ (2006) Initial experience with a commercial cone beam optical CT unit for polymer gel dosimetry II: Clinical potential. J Phys Conf Ser, 56:183-6.

16. Wuu CS and Xu Y (2011) 3-D dosimetry with optical CT scanning of polymer gels and radiochromic plastic dosimeter. Rad Meas, 46: 1903-7.

17. Cheng HW, Ho CJ, Lee CC, Tu SJ, Shih BY, Chao TC (2011) Development of a novel optical CT employing a laser to create a collimated line-source with a flat-top intensity distribution. Rad Meas, 46: 1932-5.

Int. J. Radiat. Res., Vol. 15 No. 2, April 2017 
18. Hilts M, Jirasek A, Duzenli C (2005) Technical consideration for implantation of X-ray CT polymer gel dosimetry. Phys Med Biol, 50:1727-45.

19. Sedaghat M, Zahmatkesh MH, Jaberi R, Akhlaghpoor S, Allahverdi M (2005) Brachytherapy polymer gel dosimetry with xCT. Int J Radiat Res,3(3):101-8.

20. Remo AC, Bamber JC, Partridge M, Bush NL, Webb S (2007) Characterization of the ultrasonic attenuation coefficient and its frequency dependence in a polymer gel dosimeter. Phys Med Biol, 52:6747-59.

21. Akhlaghpoor S, Zahmatkesh MH, Pourbeigi H (2003) 3D MRI gel dosimetry based on image intensity (A new approach). Int J Radiat Res, 1(1): 45-50.

22. Abtahi SM, Shahriari M, Zahmatkesh MH, Khalafi $H$, Akhlaghpoor S, Bagheri S (2008) A new approach to contrast enhancement in MAGICA gel dosimeter image with MRI technique. Int J Radiat Res, 6(3):151-6.

23. Watanabe $Y$ and Kubo $H$ (2011) A variable echo-number method for estimating R2 in MRI-based polymer gel dosimetry. Med Phys, 38 (2):975-82.

24. Azadbakht B, Zahmatkesh MH, Hadad k, Bagheri S (2008) Verification of the PAGAT polymer gel dosimeter by photon beams using magnetic resonance imaging. Int J Radiat Res, 6(2):83-7.

25. De Deene $Y$ and Baldock C (2002) Optimization of multiple spin-echo sequences for 3D polymer gel dosimetry. Phys Med Biol, 47: 3117-41.

26. Baldock C, Lepage M, Back SA, Murry PJ, Jayasekera PM, Porter D, Kron T (2001) Dose resolution in radiotherapy gel dosimetry: effect of echo spacing in MRI pulse sequence. Phys Med Biol, 46: 449-60.

27. Baustert IC, Oldham M, Smith TAD, Hayes C, Webb S, Leach MO (2000) Optimized MR imaging for polyacrylamide gel dosimetry. Phys Med Biol, 45(4): 847.

28. Seimenis I, Moutsatsos A, Petrokokkinos L, Kantemiris I, Benekos O, Efstathopoulos E, Papagiannis P, Spevacek V, Semnicka J, Dvorak P (2009) The use of high field strength and parallel imaging techniques for MRI-based gel dosimetry in stereotactic radiosurgery. J Instrum, 4(07):P07004.

29. MacDougall ND, Miquel ME, Wilson DJ, Keevil SF, Smith MA (2005) Evaluation of the dosimetric performance of BANG $3^{\circledR}$ polymer gel. Phys Med Biol, 50(8):1717.

30. Watanabe $Y$ and Kubo $H$ (2011) A variable echo-number method for estimating R2 in MRI-based polymer gel dosimetry. Med Phys, 38(2):975-82.

31. Abtahi SM, Aghamiri SMR, Khalafi H (2014) Optical and MRI investigations of an optimized acrylamide-based polymer gel dosimeter. J Radioanal Nucl Chem, 300: 287-301.
32. Absorbed dose determination in external beam radiotherapy: an International Code of Practice for dosimetry based on standards of absorbed dose to water. Vienna: 2000 Tech. Rep. Series no. 398.

33. De Deene $Y$, Van de Walle R, Achten E, De Wagter C (1998) Mathematical analysis and experimental investigation of noise in quantitative magnetic resonance imaging applied in polymer gel dosimetry. Signal Processing, 70:85-101.

34. Joaquim $P$ and Marques de S (2007) Applied Statistics Using SPSS, STATISTICA, MATLAB and R in statistical analysis applications. Springer, New York.

35. Maryanski MJ, Gore JC, Kennan RP, Schulz RJ (1993) NMR relaxation enhancement in gels polymerized and crosslinked by ionizing radiation: a new approach to 3D dosimetry by MRI. Magn Reson Im, 11:253-8.

36. JCGM (2008) Evaluation of measurement data - Guide to the expression of uncertainty in measurement (GUM)JCGM 100.2008 GUM 1995 with minor corrections.

37. IBM Corp. Released 2010. IBM SPSS Statistics for Windows, Version 19.0. Armonk, NY: IBM Corp.

38. De Deene $Y$, Hanselaer P, Wagter CD, Achten E, Neve WD ( 2000 ) An investigation of the chemical stability of a monomer/polymer gel dosimeter. Phys Med Biol, 45:85978.

39. Gholami M, Shahbazi-Gahrouei D, Allahverdi Pourfallah T (2015) Dose response evaluation of a low density anoxic polymer gel dosimeter using MRI. Int J Radiat Res, 13 (3):243-9.

40. Parwaie $W$, Yarahmadi $M$, Nedaie HA, Zahmatkesh $M H$, Barati AH, Afkhami M (2016) Evaluation of MRI-based MAGIC polymer gel dosimeter in small photon fields. Int $\mathrm{J}$ Radiat Res, 14(1):59-65.

41. Vandecasteele J and De Deene Y (2013) On the validity of 3D polymer gel dosimetry: III. MRI-related error sources. Physics in Medicine and Biology, 58(1): 63.

42. Reeder SB, Atalar E, Anthony Z, Faranesh ???, McVeigh ER (1999) Multi-Echo Segmented k-space Imaging: An Optimized Hybrid Sequence for Ultrafast Cardiac Imaging. Magn Reson Med, 41: 375-85.

43. De Deene Y (2004) Fundamentals of MRI measurements for gel dosimetry. Journal of Physics, Conference Series 3:87-114.

44. Fransson A, Ericsson A, Jung B, Sperber GO (1993) Properties of the phase-alternating phase-shift (PHAPS) multiple spin-echo protocol in MRI: a study of the effects of imperfect RF pulses. Magn Reson Im, 11 (6):771-84. 